\title{
On Basing Private Information Retrieval on NP-Hardness
}

\author{
Tianren Liu ${ }^{(凶)}$ and Vinod Vaikuntanathan \\ MIT CSAIL, Cambridge, USA \\ liutr@mit.edu, vinodv@csail.mit.edu
}

\begin{abstract}
The possibility of basing the security of cryptographic objects on the (minimal) assumption that $\mathbf{N P} \nsubseteq \mathbf{B P P}$ is at the very heart of complexity-theoretic cryptography. Most known results along these lines are negative, showing that assuming widely believed complexity-theoretic conjectures, there are no reductions from an NPhard problem to the task of breaking certain cryptographic schemes. We make progress along this line of inquiry by showing that the security of single-server single-round private information retrieval schemes cannot be based on NP-hardness, unless the polynomial hierarchy collapses. Our main technical contribution is in showing how to break the security of a PIR protocol given an SZK oracle. Our result is tight in terms of both the correctness and the privacy parameter of the PIR scheme.
\end{abstract}

\section{Introduction}

The possibility of basing the security of cryptographic objects on the (minimal) assumption that $\mathbf{N P} \nsubseteq \mathbf{B P P}$ is at the very heart of complexity-theoretic cryptography. Somewhat more precisely, "basing primitive $X$ on NP-hardness" means that there is a construction of primitive $X$ and a probabilistic polynomialtime oracle algorithm (a reduction) $R$ such that for every oracle $A$ that "breaks the security of $X^{\prime \prime}, \operatorname{Pr}\left[R^{A}(\phi)=1\right] \geq 2 / 3$ if $\phi \in$ SAT and $\operatorname{Pr}\left[R^{A}(\phi)=1\right] \leq 1 / 3$ otherwise.

There are a handful of impossibility results which show that, assuming widely believed complexity-theoretic conjectures, the security of various cryptographic objects cannot be based on NP-hardness. We discuss these results in detail in Sect. 1.2. In this work, we make progress along these lines of inquiry by showing that (single server) private information retrieval (PIR) schemes cannot be based on NP-hardness, unless the polynomial hierarchy collapses.

Main Theorem 1 (Informal). If there is a probabilistic polynomial time reduction from solving SAT to breaking a single-server, one round, private information retrieval scheme, then $\mathbf{N P} \subseteq \mathbf{c o A M}$.

T. Liu-Supported by NSF Grants CNS-1350619 and CNS-1414119.

V. Vaikuntanathan-Research supported in part by NSF Grants CNS-1350619 and CNS-1414119, Alfred P. Sloan Research Fellowship, Microsoft Faculty Fellowship, the NEC Corporation, and a Steven and Renee Finn Career Development Chair from MIT.

(C) International Association for Cryptologic Research 2016

E. Kushilevitz and T. Malkin (Eds.): TCC 2016-A, Part I, LNCS 9562, pp. 372-386, 2016.

DOI: $10.1007 / 978-3-662-49096-9 \_16$ 
Our result rules out security reductions from SAT that make black-box use of the adversary that breaks a PIR scheme. Other than being black-box in the adversary, the security reduction can be very general, in particular, it is allowed to make polynomially many adaptively chosen calls to the PIR-breaking adversary.

Our result is tight in terms of both the correctness and the privacy parameter of the PIR scheme. Namely, information-theoretically secure PIR schemes exist for those choice of parameters that are not ruled out by our result. We refer the reader to Sect. 3 for a formal statement of our result.

Private Information Retrieval. Private information retrieval (PIR) is a protocol between a database $D$ holding a string $x \in\{0,1\}^{n}$, and a user holding an index $i \in[n]$. The user wishes to retrieve the $i$-th bit $x_{i}$ from the database, without revealing any information about $i$. Clearly, the database can rather inefficiently accomplish this by sending the entire string $x$ to the user. The objective of PIR, then, is to achieve this goal while communicating (significantly) less than $n$ bits.

Chor, Goldreich, Kushilevitz and Sudan [CKGS98], who first defined PIR, also showed that non-trivial PIR schemes (with communication less than $n$ bits) require computational assumptions. Subsequently, PIR has been shown to imply one-way functions [BIKM99], oblivious transfer [CMO00] and collision-resistant hashing [IKO05], placing it in cryptomania proper.

On the other hand, there have been several constructions of PIR with decreasing communication complexity under various cryptographic assumptions [KO97, CMS99, Lip05, BGN05, GR05, Gen09, BV11].

In particular, Kushilevitz and Ostrovsky [KO97] were the first to show a construction of PIR with $O\left(n^{\epsilon}\right)$ communication (for any constant $\epsilon>0$ ) assuming the existence of additively homomorphic encryption schemes. Some of the later constructions of PIR [CMS99, Lip05, GR05, BV11] achieve polylog $(n)$ communication under number-theoretic assumptions such as the Phi-hiding assumption and the LWE assumption. Notably, all of them are single-round protocols, involving one message from the user to the server and one message back.

\subsection{Our Techniques}

The core of our proof is an attack against any single-server one-round PIR protocol given access to an SZK oracle. In particular, we show that given an oracle to the entropy difference (ED) problem, which is complete for SZK, one can break any single-server one-round PIR protocol. Once we have this result, the rest follows from a beautiful work of Mahmoody and Xiao [MX10] who show that $\mathbf{B P} \mathbf{P}^{\mathbf{S Z K}} \subseteq \mathbf{A M} \cap \mathbf{c o A M}$. That is, if there is a reduction from deciding SAT to breaking single-server one-round PIR, then SAT $\in \mathbf{B P P}^{\mathbf{S Z K}}$ and therefore, by $[\mathrm{MX10]}$, SAT $\in \mathbf{A M} \cap \mathbf{c o A M}$. In turn, from the work of Boppana, Håstad and Zachos [BHZ87], this means that the polynomial hierarchy collapses to the second level.

The intuition behind the attack against PIR protocols is simple. Assume that the database is uniformly random and the user's query is fixed. Let $X$ be a random variable that denotes the database, and let $A$ be a random variable 
that denotes the PIR answer (on input a query $q$ from a user trying to retrieve the $i$-th bit). We have two observations.

1. The answer enables the user to learn the $i$-th bit. In other words, the mutual information between the $i$-th database bit $X_{i}$ and the answer $A$ has to be large. Indeed, we show that if the PIR protocol is correct with probability $1-\varepsilon$, then this mutual information is at least $1-h(\varepsilon)$, where $h$ is the binary entropy function.

2. The answer does not contain a large amount of information about all the database entries. Indeed, the entropy of the answer is limited by its length which is much shorter than the size of the database. We show that for most indices $j$, the answer contains little information about the $j$-th bit, that is the mutual information between $A$ and $X_{j}$ is small.

We then proceed as follows. Given the user's query $q$, an efficient adversary can construct a circuit sampling from joint distribution $(X ; A)$. Armed with the entropy difference ED oracle, the adversary can estimate $I\left(X_{j} ; A\right)$ for any index $j$. Since $I\left(X_{i} ; A\right)$ is close to 1 (where $i$ is the index underlying the query $q$ ) and $I\left(X_{j} ; A\right)$ is small for most indices $j$, the adversary can predict $i$ much better than random guessing. This breaks the security of PIR.

We refer the reader to Theorem 3.1 for the formal statement, and to Proposition 2.8 which shows that the parameters of Theorem 3.1 are tight.

\subsection{Related Work}

Brassard [Bra79] showed that one-way permutations cannot be based on NPhardness. Subsequently, Goldreich and Goldwasser [GG98], in the process of clarifying Brassard's work, showed that public-key encryption schemes that satisfy certain very special properties cannot be based on NP-hardness. In particular, one of their conditions require that it should be easy to certifying an invalid key as such.

Akavia, Goldreich, Goldwasser and Moshkovitz [AGGM06], and later Bogdanov and Brzuska [BB15], showed that a special class of one-way functions called size-verifiable one-way functions cannot be based on NP-hardness. A size-verifiable one-way function, roughly speaking, is one in which the size of the set of pre-images can be efficiently approximated via an AM protocol.

Most recently, Bogdanov and Lee [BL13a] showed that (even simple) homomorphic encryption schemes cannot be based on NP-hardness. This includes additively homomorphic encryption as well as homomorphic encryption schemes that only support the majority function, as special cases. While PIR schemes can be constructed from additively homomorphic encryption, we are not aware of a way to use PIR to obtain any type of non-trivial homomorphic encryption scheme.

Several works have also explored the problem of basing average-case hardness on (worst case) NP-hardness, via restricted types of reductions, most notably non-adaptive reductions that make all its queries to the oracle simultaneously. 
The work of Feigenbaum and Fortnow, subsequently strengthened by Bogdanov and Trevisan [BT06], show that there cannot be a non-adaptive reduction from (worst-case) SAT to the average-case hardness of any problem in NP, unless $\mathbf{P H} \subseteq \boldsymbol{\Sigma}_{\mathbf{2}}$ (that is, the polynomial hierarchy collapses to the second level). In contrast, our results rule out even adaptive reductions (to much stronger primitives).

\section{Definitions}

\subsection{Information Theory Background}

A random variable $X$ over a finite set $S$ is defined by its probability mass function $p_{X}: S \rightarrow[0,1]$ such that $\sum_{x \in S} p_{X}(x)=1$. We use uppercase letters to denote random variables. The Shannon entropy of a random variable $X$, denoted $H(X)$, is defined as

$$
H(X)=\sum_{x} p_{X}(x) \log _{2} \frac{1}{p_{X}(x)} .
$$

Let $\operatorname{Bern}(p)$ denote the Bernoulli distribution on $\{0,1\}$ which assigns a probability of $p$ to 1 and $1-p$ to 0 . We will denote by $h(p)=H(\operatorname{Bern}(p))=$ $p \log _{2} \frac{1}{p}+(1-p) \log _{2} \frac{1}{1-p}$ the Shannon entropy of the distribution $\operatorname{Bern}(p)$.

Let $X$ and $Y$ be two (possibly dependent) random variables. The conditional entropy of $Y$ given $X$, denoted $H(Y \mid X)$, is defined as $H(Y \mid X)=H(X Y)-$ $H(X)$, where $X Y$ denotes the joint distribution of $X$ and $Y$. Informally, $H(Y \mid X)$ measures the (residual) uncertainty of $Y$ when $X$ is known.

The mutual information between random variables $X$ and $Y$ is

$$
I(X ; Y)=H(X)+H(Y)-H(X Y)=H(Y)-H(Y \mid X)=H(X)-H(X \mid Y)
$$

which measures the information that $X$ reveals about $Y$ (and vice versa). In particular, if two random variables $X, Y$ are independent, their mutual information is zero.

The conditional mutual information between random variables $X$ and $Y$ given $Z$, denoted $I(X ; Y \mid Z)$, is defined as

$$
I(X ; Y \mid Z)=H(X \mid Z)+H(Y \mid Z)-H(X Y \mid Z) .
$$

We will use without proof that entropy, conditional entropy, mutual information, conditional mutual information are non-negative.

We will need the following simple propositions.

Proposition 2.1. Let $X \sim \operatorname{Bern}\left(\frac{1}{2}\right)$ be a random variable uniformly distributed in $\{0,1\}$, let $N \sim \operatorname{Bern}(\varepsilon)$ be a noise that is independent from $X$, and let $\hat{X}=$ $X \oplus N$ be the noisy version of $X$. Then $I(\hat{X} ; X)=1-h(\varepsilon)$. Moreover, for any random variable $X^{\prime}$ satisfying $\operatorname{Pr}\left[X^{\prime}=X\right] \geq 1-\varepsilon$,

$$
I\left(X^{\prime} ; X\right) \geq 1-h(\varepsilon) \text {. }
$$


Proof. Clearly, $I(\hat{X} ; X)=H(X)-H(X \mid \hat{X})=1-h(\varepsilon)$. Furthermore, the random variable $\hat{X}=X \oplus N$ minimizes the mutual information $I(\hat{X} ; X)$ under the constraint that $\operatorname{Pr}[\hat{X}=X] \geq 1-\varepsilon$. In particular, we have

$$
I\left(X^{\prime} ; X\right)=H(X)-H\left(X \mid X^{\prime}\right)=1-H\left(X \oplus X^{\prime} \mid X^{\prime}\right) \geq 1-H\left(X \oplus X^{\prime}\right) \geq 1-h(\varepsilon)
$$

for any random variable $X^{\prime}$ satisfying $\operatorname{Pr}\left[X^{\prime}=X\right] \geq 1-\varepsilon$.

Proposition 2.2 (Conditioning Decreases Entropy). For any random variables $X, Y, Z$, it holds that $H(X) \geq H(X \mid Y) \geq H(X \mid Y Z)$.

In general, conditioning can increase or decrease mutual information, but when conditioning on an independent variable, mutual information increases.

Proposition 2.3 (Conditioning on Independent Variables Increases Mutual Information). For random variables $X, Y, Z$ such that $Y$ and $Z$ are independent, $I(X ; Y \mid Z) \geq I(X ; Y)$.

Proof. As $Y, Z$ are independent, $H(Y \mid Z)=H(Y)$.

$$
I(X ; Y \mid Z)=H(Y \mid Z)-H(Y \mid X Z) \geq H(Y)-H(Y \mid X)=I(X ; Y) .
$$

Proposition 2.4 (Data Processing for Mutual Information). Assume random variables $X, Y, Z$ satisfies $X \rightarrow Y \rightarrow Z$, i.e. $X$ and $Z$ are independent conditional on $Y$, then $I(X ; Y) \geq I(X ; Z)$.

Proof. Since $X$ and $Z$ are independent conditional on $Y$ (meaning $I(X ; Z \mid Y)=$ $0)$, we have $H(X \mid Y Z)=H(X \mid Y)$. Thus

$$
I(X ; Y)=H(X)-H(X \mid Y)=H(X)-H(X \mid Y Z) \geq H(X)-H(X \mid Z)=I(X ; Z) .
$$

Proposition 2.5 (Chain Rule for Mutual Information). For random variables $X_{1}, \ldots, X_{n}, Y$, it holds that

$$
I\left(X_{1} \ldots X_{n} ; Y\right)=\sum_{i=1}^{n} I\left(X_{i} ; Y \mid X_{1} \ldots X_{i-1}\right) .
$$

\subsection{Single-Server One-Round Private Information Retrieval}

In a single-server private information retrieval (PIR) protocol, the database holds $n$ bits of data $x \in\{0,1\}^{n}$. The user, given an index $i \in[n]$, would like to retrieve the $i$-th bit from the server, without revealing any information about $i$. The user does so by generating a query based on $i$ using a randomized algorithm; the server responds to the query with an answer. The user, given the answer and the randomness used to generate the query, should be able to learn the $i$-th bit $x_{i}$.

We specialize our definitions to the case of single round protocols.

Definition 2.6 (Private Information Retrieval). A single-server one round private information retrieval (PIR) scheme is a tuple (Qry, Ans, Rec) of algorithms such that 
- The query algorithm Qry is a probabilistic polynomial-time algorithm such that $\operatorname{Qry}\left(1^{n}, i\right) \rightarrow(q, \sigma)$, where $i \in[n]$. Here, $q$ is the PIR query and $\sigma$ is the secret state of the user (which, without loss of generality, is the randomness used by the algorithm).

- The answer algorithm Ans is a probabilistic polynomial-time algorithm such that $\operatorname{Ans}(x, q) \rightarrow a$, where $x \in\{0,1\}^{n}$. Let $\ell$ denote the length of the answer, i.e. $a \in\{0,1\}^{\ell}$.

- The reconstruction algorithm $\mathbf{R e c}$ is a probabilistic polynomial-time algorithm such that $\boldsymbol{\operatorname { R e c }}(a, \sigma) \rightarrow b$ where $b \in\{0,1\}$.

Correctness. A PIR scheme (Qry, Ans, Rec) is $(1-\varepsilon)$-correct if for any $x \in$ $\{0,1\}^{n}$ and for any $i$,

$$
\operatorname{Pr}\left[\operatorname{Qry}\left(1^{n}, i\right) \rightarrow(q, \sigma), \operatorname{Ans}(x, q) \rightarrow a: \operatorname{Rec}(a, \sigma)=x_{i}\right] \geq 1-\varepsilon(n)
$$

where the probability is taken over the random tapes of Qry, Ans, Rec. We call $\epsilon$ the error probability of the PIR scheme.

Privacy. The standard definition of computational privacy for PIR requires that the database cannot efficiently distinguish between queries for different indices. Formally, a PIR scheme is $\delta$-IND-secure (for some $\delta=\delta(n)$ ) if for any probabilistic polynomial-time algorithm $\mathcal{A}=\left(\mathcal{A}_{1}, \mathcal{A}_{2}\right)$, there exists a negligible function $\delta$ such that

$$
\operatorname{Pr}\left[\begin{array}{c}
\mathcal{A}_{1}\left(1^{n}\right) \rightarrow\left(i_{0}, i_{1}, \tau\right) \\
b \stackrel{\$}{\leftarrow}\{0,1\} \\
\operatorname{Qry}\left(1^{n}, i_{b}\right) \rightarrow(q, \sigma) \\
\mathcal{A}_{2}\left(1^{n}, q, \tau\right) \rightarrow b^{\prime}
\end{array}: b^{\prime}=b\right]<\frac{1}{2}+\delta(n)
$$

(Here and in the sequel, $\tau$ will denote the state that $\mathcal{A}_{1}$ passes on to $\mathcal{A}_{2}$ ).

The adversary in this privacy definition is interactive, which introduces difficulties in defining an oracle that breaks PIR. To make our task easier, we consider an alternative, non-interactive definition which is equivalent to (1).

We call a PIR scheme $\delta$-GUESS-secure if for any probabilistic polynomialtime algorithm $\mathcal{A}$, there exists a negligible function $\delta$ such that

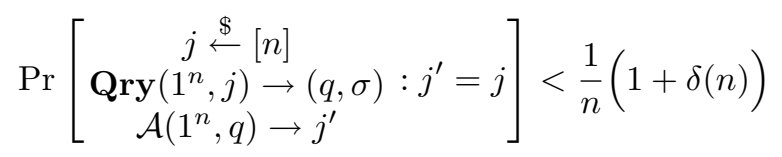

These two definitions of privacy are equivalent up to a polynomial factor in $n$, as we show in the next proposition.

Proposition 2.7. If a PIR scheme is $\delta_{1}$-IND-secure (according to Definition (1)), then it is $\delta_{2}$-GUESS-secure (according to Definition (2)) where $\delta_{2}=n \delta_{1}$. Similarly, if a PIR scheme is $\delta_{2}$-GUESS-secure, then it is $\delta_{1}$-IND-secure where $\delta_{1}=\delta_{2} / 2$. 
Proof. Assume that a probabilistic polynomial-time (p.p.t.) adversary algorithm $\mathcal{A}$ breaks $\delta_{2}$-privacy according to Definition (2). We construct an adversary $\mathcal{B}=$ $\left(\mathcal{B}_{1}, \mathcal{B}_{2}\right)$ that breaks Definition (1).

The algorithm $\mathcal{B}_{1}\left(1^{n}\right)$ picks two random indices $i_{0}$ and $i_{1}$ and outputs $i_{0}, i_{1}$ and $\tau=\left(i_{0}, i_{1}\right)$, algorithm $\mathcal{B}_{2}\left(1^{n}, q, \tau=\left(i_{0}, i_{1}\right)\right)$ calls $\mathcal{A}\left(1^{n}, q\right)$ to get an index $i$, and outputs 0 if and only if $i=i_{0}$. Then,

$$
\begin{aligned}
& \operatorname{Pr}\left[\begin{array}{c}
\mathcal{B}_{1}\left(1^{n}\right) \rightarrow\left(i_{0}, i_{1}, \tau\right) \\
b \stackrel{\$}{\leftarrow}\{0,1\} \\
\operatorname{Qry}\left(1^{n}, i_{b}\right) \rightarrow(q, \sigma) \\
\mathcal{B}_{2}\left(1^{n}, q, \tau\right) \rightarrow b^{\prime}
\end{array}: b^{\prime}=b\right]=\operatorname{Pr}\left[\begin{array}{cc}
i_{0}, i_{1} \stackrel{\$}{\leftarrow}[n] & i=i_{0}, b=0 \\
b \stackrel{\$}{\leftarrow}\{0,1\} & \text { or } \\
\operatorname{Qry}\left(1^{n}, i_{b}\right) \rightarrow(q, \sigma) & i \neq i_{0}, b \neq 0 \\
\mathcal{A}\left(1^{n}, q\right) \rightarrow i &
\end{array}\right] \\
& =\frac{1}{2} \operatorname{Pr}\left[\begin{array}{c}
i_{0}, i_{1} \stackrel{\$}{\leftarrow}[n] \\
\operatorname{Qry}\left(1^{n}, i_{0}\right) \rightarrow(q, \sigma): i=i_{0} \\
\mathcal{A}\left(1^{n}, q\right) \rightarrow i
\end{array}\right]+\frac{1}{2} \operatorname{Pr}\left[\begin{array}{c}
i_{0}, i_{1} \stackrel{\$}{\leftarrow}[n] \\
\operatorname{Qry}\left(1^{n}, i_{1}\right) \rightarrow(q, \sigma): i \neq i_{0} \\
\mathcal{A}\left(1^{n}, q\right) \rightarrow i
\end{array}\right] \\
& \geq \frac{1}{2} \frac{1}{n}\left(1+\delta_{2}(n)\right)+\frac{1}{2}\left(1-\frac{1}{n}\right)=\frac{1}{2}\left(1+\frac{\delta_{2}(n)}{n}\right)
\end{aligned}
$$

Thus, $\left(\mathcal{B}_{1}, \mathcal{B}_{2}\right)$ breaks $\frac{\delta_{2}}{n}$-privacy according to Definition (1).

In the other direction, assume that a p.p.t. adversary algorithm $\mathcal{A}=\left(\mathcal{A}_{1}, \mathcal{A}_{2}\right)$ breaks $\delta_{1}$-privacy according to Definition (1). We construct an adversary $\mathcal{B}$ that works as follows. $\mathcal{B}$ runs $\mathcal{A}_{1}$ to get $\left(i_{0}, i_{1}, \tau\right) \leftarrow \mathcal{A}_{1}\left(1^{n}\right)$, gets a challenge query $q$ and runs $\mathcal{A}_{2}$ to get $b \leftarrow \mathcal{A}_{2}\left(1^{n}, q, \tau\right)$. $\mathcal{B}$ simply outputs $i_{b}$. Then, we have:

$$
\begin{aligned}
& \operatorname{Pr}\left[\begin{array}{c}
j \stackrel{\$}{\leftarrow}[n] \\
\left.\operatorname{Qry}\left(1^{n}, j\right) \rightarrow(q, \sigma): j^{\prime}=j\right] \\
\mathcal{B}\left(1^{n}, q\right) \rightarrow j^{\prime}
\end{array}\right]=\operatorname{Pr}\left[\begin{array}{c}
\mathcal{A}_{1}\left(1^{n}\right) \rightarrow\left(i_{0}, i_{1}, \tau\right) \\
j \stackrel{\$}{\leftarrow}[n] \\
\operatorname{Qry}\left(1^{n}, j\right) \rightarrow(q, \sigma) \\
\mathcal{A}_{2}\left(1^{n}, q, \tau\right) \rightarrow b
\end{array}: j=i_{b}\right] \\
& \left.=\frac{2}{n} \operatorname{Pr}\left[\begin{array}{c}
\mathcal{A}_{1}\left(1^{n}\right) \rightarrow\left(i_{0}, i_{1}, \tau\right) \\
j \stackrel{\$}{\leftarrow}\left\{i_{0}, i_{1}\right\} \\
\operatorname{Qry}\left(1^{n}, j\right) \rightarrow(q, \sigma) \\
\mathcal{A}_{2}\left(1^{n}, q, \tau\right) \rightarrow b
\end{array}\right] j=i_{b}\right] \geq \frac{2}{n}\left(\frac{1}{2}+\delta_{1}(n)\right)=\frac{1}{n}\left(1+2 \delta_{1}(n)\right)
\end{aligned}
$$

Thus, $\mathcal{B}$ breaks $2 \delta_{1}$-privacy according to Definition (2).

Answer Communication Complexity. We define the answer communication complexity of the PIR scheme to be the number of bits in the server's response to a PIR query. (This is denoted by $\ell$ in Definition 2.6). Similarly, we call the bitlength of the query as the query communication complexity, and their sum as the total communication complexity. In this work, we are interested in PIR protocols with a "small" answer communication complexity (regardless of their query communication complexity). Since our main result is a lower bound, this only makes it stronger.

Typically, we are interested in PIR schemes with answer communication complexity $\ell=o(n)$. Otherwise, e.g. when $\ell=n$, there is a trivial PIR protocol with perfect privacy, where the user sends nothing and the server sends the whole database $x$. The following proposition shows a tradeoff between the correctness error and answer communication complexity of perfectly private PIR schemes. 
Proposition 2.8. There exists a PIR scheme with perfect information-theoretic privacy, error probability $\varepsilon$, and answer communication complexity $\ell=n \cdot(1-$ $\left.h(\varepsilon)+O\left(n^{-1 / 4}\right)\right)$.

Consider a PIR scheme where the user sends nothing and the server sends the whole database to the user, incurring an answer communication complexity of $n$ bits. The query contains no information about the index $i$, and this achieves perfect privacy and correctness. The idea is that given the possibility of a correctness error of $\varepsilon$, the server can compress the database into $\ell<n$ bits, such that the user can still recover the database with at most $\varepsilon$ error.

This is a fundamental problem in information theory, called "lossy source coding" [Sha59]. Let $X$ be a uniform random Bernoulli variable. Proposition 2.1 says that for any random variable $\hat{X}$ such that $\operatorname{Pr}[\hat{X}=X] \geq 1-\varepsilon, I(\hat{X}, X) \geq$ $1-h(\varepsilon)$. Therefore, to compress a random binary string and to recover the string from the lossy compression with $(1-\varepsilon)$ accuracy, the compression ratio need to be at least $1-h(\varepsilon)$.

There exists a lossy source coding scheme almost achieves the information theoretical bound [Ari09, KU10], i.e., when $\ell=n \cdot\left(1-h(\varepsilon)+O\left(n^{-1 / 4}\right)\right)$, there exists efficient algorithms $E:\{0,1\}^{n} \rightarrow\{0,1\}^{\ell}$ and $D:\{0,1\}^{\ell} \rightarrow\{0,1\}^{n}$, such that for randomly chosen $X \in\{0,1\}^{n}$ and for any index $i \in[n]$,

$$
\underset{X}{\operatorname{Pr}}\left[\hat{X}=D(E(X)): \hat{X}_{i}=X_{i}\right] \geq 1-\varepsilon .
$$

Therefore, if the server sends $E(x)$ as the answer, then the PIR scheme achieves $(1-\varepsilon)$ correctness on a random database. Moreover, we can extend this to work for any database by the following scheme which has a query communication complexity of $n$ bits and an answer communication complexity of $\ell$ bits.

- User sends a query $m$, which is a random string in $\{0,1\}^{n}$;

- Server answers by $a=E(m \oplus x)$;

- User retrieves the whole database by $\hat{x}=D(a) \oplus m$.

Then for any database and any index $i \in[n], \operatorname{Pr}\left[\hat{x}_{i}=x_{i}\right] \geq 1-\varepsilon$.

Reduction to Breaking PIR. What does it mean for a reduction to decide a language $L$ assuming that there is a p.p.t. adversary that breaks PIR? For any language $L$, we say $L$ can be reduced to breaking the $\delta$-GUESS-security of PIR scheme (Qry, Ans, Rec) if there exists a probabilistic polynomial-time oracle Turing machine (OTM) $M$ such that for all $x$ and for all "legal" oracles $\mathcal{O}_{\delta}^{\text {PIR }}$,

$$
\begin{array}{ll}
\operatorname{Pr}\left[M^{\mathcal{O}}{ }_{\delta}^{\text {PIR }}(x)=1\right] \geq 2 / 3 & \text { if } x \in L \\
\operatorname{Pr}\left[M^{\mathcal{O}_{\delta}^{\text {PIR }}}(x)=1\right] \leq 1 / 3 & \text { if } x \notin L
\end{array}
$$

where the probability is taken over the coins of the machine $M$ and the oracle $\mathcal{O}_{\delta}^{\text {PIR }}$. We stress that $M$ is allowed to make adaptive queries to the oracle. 
By a legal $\delta$-breaking oracle $\mathcal{O}_{\delta}^{\text {PIR }}$, we mean one that satifies

$$
\operatorname{Pr}\left[\begin{array}{c}
j \leftarrow[n] \\
\operatorname{Qry}\left(1^{n}, j\right) \rightarrow(q, \sigma): j=j^{\prime} \\
\mathcal{O}_{\delta}^{\operatorname{PIR}}(q) \rightarrow j^{\prime}
\end{array}\right] \geq \frac{1}{n}(1+\delta)
$$

where the probability is taken over the coins used in the experiment, including those of Qry and $\mathcal{O}_{\delta}^{\text {PIR }}$.

\subsection{Entropy Difference}

Entropy Difference (ED) is a promise problem that is complete for SZK [GV99]. Entropy Difference is a promise problem defined as

- YES instances: $(X, Y)$ such that $H(X) \geq H(Y)+1$

- NO instances: $(X, Y)$ such that $H(Y) \geq H(X)+1$

where $X$ and $Y$ are distributions encoded as circuits which sample from them.

We list a few elementary observations regarding the power of an oracle that decides the entropy difference problem.

First, given an entropy difference oracle, a polynomial-time algorithm can distinguish between two distributions $X$ and $Y$ such that either $H(X) \geq H(Y)+$ $\frac{1}{s}$ or $H(Y) \geq H(X)+\frac{1}{s}$ for any polynomial function $s$. That is, one can solve the entropy difference problem up to any inverse-polynomial precision. This can be done as follows: For distributions $X, Y$, we query the Entropy Difference oracle with $\left(X_{1} \ldots X_{s}, Y_{1} \ldots Y_{s}\right)$, where $X_{i} \sim X, Y_{i} \sim Y$ and $X_{1}, \ldots, X_{s}$ are i.i.d. and $Y_{1}, \ldots, Y_{s}$ are i.i.d. Then we would be able to distinguish between $H(X) \geq H(Y)+\frac{1}{s}$ and $H(Y) \geq H(X)+\frac{1}{s}$.

Similarly, a polynomial-time algorithm can use the Entropy Difference oracle to distinguish between $H(X) \geq \hat{h}+\frac{1}{s}$ and $H(X) \leq \hat{h}-\frac{1}{s}$ for a given $\hat{h}$. This can be done as follows: construct a distribution $Y$ that $2 s \hat{h}-1<H(Y)<2 s \hat{h}+1$ and query the Entropy Difference oracle with the distributions $X_{1} \ldots X_{2 s}$ and $Y$, where $X_{1}, \ldots, X_{2 s}$ are independent copies of $X$. Therefore, a polynomialtime algorithm given Entropy Difference oracle can estimate $H(X)$ to within any additive inverse-polynomial precision by binary search.

Finally, assume that $X$ and $Y$ are random variables encoded as a circuit which samples from their joint distributions. Then, a polynomial-time algorithm given an Entropy Difference oracle can also estimate the conditional entropy $H(X \mid Y)$, mutual information $I(X ; Y)$ to any inverse-polynomial precision. Here the precision is measured by absolute additive error.

\section{$3 \quad$ PIR and NP-Hardness}

Theorem 3.1 (Main Theorem). Let $\Pi=($ Qry, Ans, Rec) be any $(1-\epsilon)$ correct PIR scheme with $n$-bit databases and answer communication complexity $\ell$. Let $L$ be any language. If 
1. there exists a reduction from $L$ to breaking the $\delta$-privacy of $\Pi$ in the sense of Equation (2); and

2. there is a polynomial $p(n)$ such that

$$
\ell \cdot(1+\delta) \leq n \cdot(1-h(\varepsilon))-1 / p(n)
$$

\section{then $L \in \mathbf{A M} \cap \mathbf{c o A M}$.}

In particular, using the result of [BHZ87], this tells us that unless the polynomial hierarchy collapses, there is no reduction from SAT to breaking the privacy of a PIR scheme with parameters as above.

We note that the bound in the lemma is tight. As Proposition 2.8 shows, there is in fact a perfectly (information-theoretically) private PIR protocol with a matching answer communication complexity of $n \cdot(1-h(\varepsilon))+o(n)$.

We prove our main theorem by combining the following two lemmas. The first lemma is our main ingredient, and says that if there is a reduction from deciding a language $L$ to breaking a PIR scheme, and the PIR scheme has a low answer communication complexity, then $L$ can be reduced to the entropy difference problem (defined in Sect. 2.3).

Lemma 3.2 $\left(\mathbf{B P P}^{\mathcal{O}_{\delta}^{\mathrm{PlR}}} \subseteq \mathbf{B P P}^{\mathrm{ED}}\right)$. Let $\Pi=(\mathbf{Q r y}, \mathbf{A n s}, \mathbf{R e c})$ be any $(1-\epsilon)$ correct PIR scheme with answer communication complexity $\ell$ and let $L$ be any language. If there exists a reduction from $L$ to $\delta$-breaking the privacy of a PIR protocol such that

$$
\frac{1-h(\varepsilon)}{\ell}-\frac{1+\delta}{n} \geq \frac{1}{p(n)}
$$

for some polynomial function $p(n)$, then there exists a probabilistic polynomial time reduction from $L$ to $\mathrm{ED}$.

As noted in Proposition 2.8, this condition is tight as there exists a PIR scheme achieving perfect privacy $(\delta=0)$ if $\ell \approx n \cdot(1-h(\varepsilon))$.

The next lemma, originally shown in [MX10] and used in [BL13b], states that any language decidable by a randomized oracle machine with access to an entropy difference oracle is in $\mathbf{A M} \cap \mathbf{c o A M}$.

Lemma 3.3 $\left(\mathrm{BPP}^{\mathrm{ED}} \subseteq \mathrm{AM} \cap \mathbf{c o A M}[\mathrm{MX10}]\right)$. For any language $L$, if there exists an OTM $M$ such that for any oracle $\mathcal{O}$ solving entropy difference

$$
\begin{array}{ll}
\operatorname{Pr}\left[M^{\mathcal{O}}(x)=1\right] \geq 2 / 3 & \text { if } x \in L \\
\operatorname{Pr}\left[M^{\mathcal{O}}(x)=1\right] \leq 1 / 3 & \text { if } x \notin L,
\end{array}
$$

then $L \in \mathbf{A M} \cap \mathbf{c o A M}$.

\subsection{Proof of the Main Theorem}

Assume that there exists a reduction from deciding a language $L$ to breaking PIR with parameters as stated in Theorem 3.1. In other words, there is a reduction from $L$ to $\delta$-breaking PIR where 


$$
\frac{1}{n}(1+\delta) \leq \frac{1-h(\varepsilon)}{\ell}-\frac{1}{n \cdot \ell \cdot p(n)}
$$

where the inequality is using the hypothesis in Theorem 3.1 that $\ell \cdot(1+\delta) \leq$ $n \cdot(1-h(\varepsilon))-1 / p(n)$.

Then, by Lemma 3.2, there is a reduction from deciding $L$ to solving the entropy difference problem ED. Combined with Lemma 3.3, we deduce that $L \in$ $\mathrm{AM} \cap \mathbf{c o A M}$.

\subsection{Proof of Lemma 3.2}

We start with two claims that are central to our proof. The first claim says that because of $(1-\varepsilon)$-correctness of the PIR scheme, the PIR answer $a$ on a query $q \leftarrow \operatorname{Qry}\left(1^{n}, i\right)$ has to contain information about the $i^{\text {th }}$ bit of the database $x_{i}$.

Claim. Let $\Pi=($ Qry, Ans, Rec) be a PIR scheme which is $(1-\varepsilon)$-correct. Fix any index $i \in[n]$. Let $X$ denote a random $n$-bit database; $(Q, \Sigma) \leftarrow \operatorname{Qry}\left(1^{n}, i\right)$; and $A \leftarrow \operatorname{Ans}(X, Q)$. Then,

$$
I\left(A ; X_{i} \mid Q\right) \geq 1-h(\varepsilon) .
$$

Proof. Define the random variable $\hat{X}_{i} \leftarrow \boldsymbol{R e c}(A, \Sigma)$. Since the PIR scheme is $(1-\varepsilon)$-correct, $\operatorname{Pr}\left[\hat{X}_{i}=X_{i}\right] \geq 1-\varepsilon$. Since $X_{i}$ is a uniform Bernoulli variable, we know from Proposition 2.1 that $I\left(\hat{X}_{i} ; X_{i}\right) \geq 1-h(\varepsilon)$.

As $X_{i}$ is independent from $Q$, we know from Proposition 2.3 that

$$
I\left(\hat{X}_{i} ; X_{i} \mid Q\right) \geq I\left(\hat{X}_{i} ; X_{i}\right) .
$$

Next, we claim that conditioning on $Q$, we have $X_{i} \rightarrow A \rightarrow \hat{X}_{i}$, in other words, $I\left(X_{i} ; \hat{X}_{i} \mid A, Q\right)=0$. This is because when $A$ and $Q$ are given, one can sample a random $\Sigma$ consistent with $Q$, then compute $\hat{X}_{i}$ from $\Sigma$ and $A$, with no knowledge of $X_{i}$. Now, Proposition 2.4 (data processing inequality for mutual information) shows that $I\left(A ; X_{i} \mid Q\right) \geq I\left(\hat{X}_{i} ; X_{i} \mid Q\right)$.

Combining what we have,

$$
I\left(A ; X_{i} \mid Q\right) \geq I\left(\hat{X}_{i} ; X_{i} \mid Q\right) \geq I\left(\hat{X}_{i} ; X_{i}\right) \geq 1-h(\varepsilon) .
$$

This completes the proof.

Claim. Let $\Pi=$ (Qry, Ans, Rec) be a PIR scheme with an answer communication complexity of $\ell$ bits. Let $X$ denote a random $n$-bit database; $(Q, \Sigma) \leftarrow \operatorname{Qry}\left(1^{n}, i\right)$; and $A \leftarrow \operatorname{Ans}(X, Q)$. Then, for any potential query $q$,

$$
\sum_{j=1}^{n} I\left(A ; X_{j} \mid Q=q\right) \leq \ell .
$$

Proof. Recall that, by definition,

$$
I\left(A ; X_{i} \mid Q\right)=\underset{Q}{\mathbb{E}}\left[I\left(A ; X_{i} \mid Q\right)\right]=\sum_{q} I\left(A ; X_{i} \mid Q=q\right) \operatorname{Pr}[Q=q]
$$


For any potential query $q$, the event $Q=q$ is independent from $X$. In particular, for any index $j$, random variable $X_{j}$ is independent from $X_{1} \ldots X_{j-1}$ given $Q=q$. So for any $q$,

$$
\begin{aligned}
\sum_{j=1}^{n} I\left(A ; X_{j} \mid Q=q\right) & \leq \sum_{j=1}^{n} I\left(A ; X_{j} \mid X_{1} \ldots X_{j-1}, Q=q\right) \\
& =I\left(A ; X_{1} \ldots X_{n} \mid Q=q\right) \\
& \leq H(A \mid Q=q) \leq \ell
\end{aligned}
$$

where the first inequality is implied by the Proposition 2.3 and the second equality is Proposition 2.5 (chain rule for mutual information).

Equations (4) and (5) are the core of the proof of Lemma 3.2. Equation (4) shows that, when retrieving the $i$-th bit, the mutual information between $X_{i}$ and server's answer $A$ is large. Equation (5) shows that, the sum of mutual information between each bit $X_{j}$ and server's answer $A$ is bounded by the answer communication complexity. Therefore, if we could measure the mutual information by an Entropy Difference oracle, we would have a pretty good knowledge of $i$.

In particular, we proceed as follows. Assume language $L$ can be solved by a probabilistic polynomial-time oracle Turing machine $\mathcal{M}$ given any oracle $\mathcal{O}_{\delta}^{\text {PIR }}$ that breaks the $\delta$-GUESS-security of the PIR scheme (Qry, Ans, Rec) where

$$
\frac{1+\delta}{n} \leq \frac{1-h(\varepsilon)}{\ell}-\frac{1}{p(n)}
$$

where $p(\cdot)$ is a fixed polynomial. We construct an efficient oracle algorithm (see Algorithm 1) that solves $L$ given an Entropy Difference oracle $\mathcal{O}^{\text {ED }}$.

For any query $q$ and index $i$, when $\mathcal{O}_{\delta}^{\mathrm{PIR}}(q)$ is simulated,

$$
\begin{aligned}
\operatorname{Pr}\left[\hat{i} \leftarrow \mathcal{O}_{\delta}^{\mathrm{PIR}}(q)\right. & : \hat{i}=i]=\frac{\hat{\mu}_{i}}{\sum_{j} \hat{\mu}_{j}} \geq \frac{\mu_{i}-\frac{1}{2 n \cdot p(n)}}{\sum_{j} \mu_{j}+\frac{1}{2 p(n)}} \\
& \geq \frac{\mu_{i}-\frac{1}{2 p(n)}}{\ell+\frac{1}{2 p(n)}} \geq \frac{\mu_{i}}{\ell} \frac{1-\frac{1}{2 p(n)}}{1+\frac{1}{2 p(n)}} \geq \frac{\mu_{i}}{\ell}\left(1-\frac{1}{p(n)}\right) \geq \frac{\mu_{i}}{\ell}-\frac{1}{p(n)}
\end{aligned}
$$

Assuming $q$ is generated from $q \leftarrow \operatorname{Qry}\left(1^{n}, i\right)$, then $\mathbb{E}\left[\mu_{i}\right]=I\left(X_{i} ; A \mid Q\right) \geq 1-$ $h(\varepsilon)$. So

$$
\begin{aligned}
\operatorname{Pr}[q & \left.\leftarrow \operatorname{Qry}\left(1^{n}, i\right), \hat{i} \leftarrow \mathcal{O}_{\delta}^{\mathrm{PIR}}(q): \hat{i}=i\right] \\
& =\underset{q \leftarrow \operatorname{Qry}\left(1^{n}, i\right)}{\mathbb{E}}[\operatorname{Pr}[\hat{i}=i \mid Q=q]] \\
& \geq \underset{q \leftarrow \operatorname{Qry}\left(1^{n}, i\right)}{\mathbb{E}}\left[\frac{\mu_{i}}{\ell}-\frac{1}{p(n)}\right] \\
& =\frac{\mathbb{E}_{q \leftarrow \operatorname{Qry}\left(1^{n}, i\right)}\left[\mu_{i}\right]}{\ell}-\frac{1}{p(n)}
\end{aligned}
$$




\section{Algorithm 1. Solving $L$ given ED oracle on input $x$ \\ 1. Simulate $\mathcal{M}^{\mathcal{O}_{\delta}^{\text {PIR }}}(x)$ \\ 2. Whenever $\mathcal{M}$ queries $\mathcal{O}_{\delta}^{\text {PIR }}(q)$, do the following:}

(a) For each index $j=1, \ldots, n$, use the entropy difference oracle to estimate

$$
\mu_{j}=I\left(A ; X_{j} \mid Q=q\right)
$$

to $\frac{1}{2 n \cdot p(n)}$ precision. More precisely, construct a circuit $C=C_{q, j}$ such that

$$
C_{q, j}(x, r)=\left(x_{j}, \operatorname{Ans}(x, q, r)\right)
$$

and estimate the mutual information between the two components of $C$ 's output. Let $\hat{\mu}_{j} \in[0,1]$ denote the estimation.

(b) Sample a random value $\hat{i} \in[n]$ according to probability distribution $p(\hat{i})=$ $\hat{\mu}_{\hat{i}} / \sum_{j} \hat{\mu}_{j}$

(c) Answer $\mathcal{M}$ 's query by $\hat{i}$

3. Output what $\mathcal{M}$ output

$$
\begin{aligned}
& \geq \frac{1-h(\varepsilon)}{\ell}-\frac{1}{p(n)} \\
& \geq \frac{1}{n}(1+\delta)
\end{aligned}
$$

\section{Discussion and Open Questions}

We show that any non-trivial single-server single-round PIR scheme can be broken in SZK. Since languages that can be decided with (adaptive) oracle access to $\mathbf{S Z K}$ live in $\mathbf{A M} \cap \mathbf{c o A M}$, this shows that there cannot be a reduction from SAT to SZK, and therefore also from SAT to breaking single-server single-round PIR.

The crucial underlying feature of single-round PIR schemes that we use is the ability to "re-randomize". By this, we mean that given a user query $q$ for an index $i$, one can generate not just a single transcript, but the distribution over all transcripts where the database is uniformly random and the prefix of the transcript is $q$. This ability to generate a transcript distribution of the same index and random database allows the adversary to break a PIR scheme with an SZK oracle.

Indeed, this is reminiscent of the work of Bogdanov and Lee who show that breaking homomorphic encryption is not NP-hard [BL13b]. Their main contribution is to show that any homomorphic encryption (whose homomorphic evaluation process produces a ciphertext that is statistically close to a fresh encryption) can be turned into a (weakly) re-randomizable encryption scheme. Once this is done, an SZK oracle can be used to break the scheme in much the same way as we do. 
A natural question arising from our work is to extend our results to multiround PIR. The key technical difficulty that arises is in sampling a random "continuation" of a partial transcript. We conjecture that our lower bound can nevertheless be extended to the multi-round case, and leave this as an interesting open problem.

Acknowledgments. We would like to thank the anonymous TCC reviewers for their careful reading and excellent suggestions, and Jayadev Acharya for valuable comments about lossy source coding and polar codes.

\section{References}

[AGGM06] Akavia, A., Goldreich, O., Goldwasser, S., Moshkovitz, D.: On basing oneway functions on NP-hardness. In: Kleinberg, J.M. (ed.) Proceedings of the 38th Annual ACM Symposium on Theory of Computing, Seattle, WA, USA, 21-23 May 2006, pp. 701-710. ACM (2006)

[Ari09] Arikan, E.: Channel polarization: a method for constructing capacityachieving codes for symmetric binary-input memoryless channels. IEEE Trans. Inf. Theory 55(7), 3051-3073 (2009)

[BB15] Bogdanov, A., Brzuska, C.: On basing size-verifiable one-way functions on NP-hardness. In: Dodis, Y., Nielsen, J.B. (eds.) TCC 2015, Part I. LNCS, vol. 9014, pp. 1-6. Springer, Heidelberg (2015)

[BGN05] Boneh, D., Goh, E.-J., Nissim, K.: Evaluating 2-DNF formulas onciphertexts. In: Kilian [Kil05], pages 325-341

[BHZ87] Boppana, R.B., Håstad, J., Zachos, S.: Does co-NP have short interactive proofs? Inf. Process. Lett. 25(2), 127-132 (1987)

[BIKM99] Beimel, A., Ishai, Y., Kushilevitz, E., Malkin, T.: One-way functions are essential for single-server private information retrieval. In: Vitter, J.S., Larmore, L.L., Leighton, F.T. (eds.) Proceedings of the ThirtyFirst Annual ACM Symposium on Theory of Computing, 1-4 May 1999, Atlanta, Georgia, USA, pp. 89-98. ACM (1999)

[BL13a] Bogdanov, A., Lee, C.H.: Limits of provable security for homomorphic encryption. In: Canetti, R., Garay, J.A. (eds.) CRYPTO 2013, Part I. LNCS, vol. 8042, pp. 111-128. Springer, Heidelberg (2013)

[BL13b] Bogdanov, A., Lee, C.H.: Limits of provable security for homomorphic encryption. In: Canetti, R., Garay, J.A. (eds.) CRYPTO 2013, Part I. LNCS, vol. 8042, pp. 111-128. Springer, Heidelberg (2013)

[Bra79] Brassard, G.: Relativized cryptography. In: 20th Annual Symposium on Foundations of Computer Science, San Juan, Puerto Rico, 29-31 October 1979, pp. 383-391. IEEE Computer Society (1979)

[BT06] Bogdanov, A., Trevisan, L.: On worst-case to average-case reductions for NP problems. SIAM J. Comput. 36(4), 1119-1159 (2006)

[BV11] Brakerski, Z., Vaikuntanathan, V.: Efficient fully homomorphic encryption from (standard) LWE. In: Ostrovsky, R. (ed.) IEEE 52nd Annual Symposium on Foundations of Computer Science, FOCS 2011, Palm Springs, CA, USA, 22-25 October 2011, pages 97-106. IEEE Computer Society (2011)

[CKGS98] Chor, B., Kushilevitz, E., Goldreich, O., Sudan, M.: Private information retrieval. J. ACM 45(6), 965-981 (1998) 
[CMO00] Di Crescenzo, G., Malkin, T., Ostrovsky, R.: Single database private information retrieval implies oblivious transfer. In: Preneel, B. (ed.) EUROCRYPT 2000. LNCS, vol. 1807, pp. 122-138. Springer, Heidelberg (2000)

[CMS99] Cachin, C., Micali, S., Stadler, M.A.: Computationally private information retrieval with polylogarithmic communication. In: Stern, J. (ed.) EUROCRYPT 1999. LNCS, vol. 1592, pp. 402-414. Springer, Heidelberg (1999)

[Gen09] Gentry, C.: Fully homomorphic encryption using ideal lattices. In: Mitzenmacher, M. (ed.) Proceedings of the 41st Annual ACM Symposium on Theory of Computing, STOC 2009, Bethesda, MD, USA, 31 May-2 June 2009, pp. 169-178. ACM (2009)

[GG98] Goldreich, O., Goldwasser, S.: On the possibility of basing cryptography on the assumption that $p \neq n p$. IACR Cryptology ePrint Archive, 1998, 5 (1998)

[GR05] Gentry, C., Ramzan, Z.: Single-database private information retrieval with constant communication rate. In: Caires, L., Italiano, G.F., Monteiro, L., Palamidessi, C., Yung, M. (eds.) ICALP 2005. LNCS, vol. 3580, pp. 803815. Springer, Heidelberg (2005)

[GV99] Goldreich, O., Vadhan, S.: Comparing entropies in statistical zero knowledge with applications to the structure of SZK. In: 1999 Proceedings of the Fourteenth Annual IEEE Conference on Computational Complexity, pp. 54-73. IEEE (1999)

[IKO05] Ishai, Y., Kushilevitz, E., Ostrovsky, R.: Sufficient conditionsfor collisionresistant hashing. In: Kilian [Kil05], pp. 445-456

[Kil05] Kilian, J. (ed.): TCC 2005. LNCS, vol. 3378. Springer, Heidelberg (2005)

[KO97] Kushilevitz, E., Ostrovsky, R.: Replication is NOT needed: SINGLE database, computationally-private information retrieval. In: 38th Annual Symposium on Foundations of Computer Science, FOCS 1997, Miami Beach, Florida, USA, 19-22 October 1997, pp. 364-373. IEEE Computer Society (1997)

[KU10] Korada, S.B., Urbanke, R.L.: Polar codes are optimal for lossy source coding. IEEE Trans. Inf. Theor. 56(4), 1751-1768 (2010)

[Lip05] Lipmaa, H.: An oblivious transfer protocol with log-squared communication. In: Zhou, J., López, J., Deng, R.H., Bao, F. (eds.) ISC 2005. LNCS, vol. 3650, pp. 314-328. Springer, Heidelberg (2005)

[MX10] Mahmoody, M., Xiao, D.: On the power of randomized reductions and the checkability of sat. In: 2010 IEEE 25th Annual Conference on Computational Complexity (CCC), pp. 64-75. IEEE (2010)

[Sha59] Shannon, C.E.: Coding theorems for a discrete source with a fidelity criterion. IRE Nat. Conv. Rec. 4(142-163), 1 (1959) 